\title{
Interpersonal synchrony affects performers' sense of agency
}

Paul Reddish $^{\mathrm{a}^{*}}$, Eddie M. W. Tong ${ }^{\mathrm{a}}$, Jonathan Jong ${ }^{\mathrm{b}}$, Harvey Whitehouse ${ }^{\mathrm{c}}$

${ }^{a}$ Department of Psychology, National University of Singapore, Singapore; ${ }^{b}$ Centre for Research in Psychology, Behavior, and Achievement, Coventry University, Coventry, United Kingdom; 'Institute of Cognitive and Evolutionary Anthropology, University of Oxford, Oxford, United Kingdom

*Corresponding author: Department of Psychology, National University of Singapore, Block AS4, 9 Arts Link, Singapore 117570; email: paulreddishnz@gmail.com.

Running head: Synchrony and agency

Total Word Count (including abstract, references, tables, captions): 11501

Main Text Word Count (excluding abstract, references, tables, captions): 9509 


\section{Interpersonal synchrony affects performers' sense of agency}

We investigated if interpersonal synchrony can lead to a sense of agency over another's movement (extended self-agency). In Experiment 1, we found that extended self-agency was greater during synchrony than asynchrony. However, we also found that synchrony boosted participants' sense that the other performer had agency over their actions (extended other-agency). This finding may have been because synchrony created a sense of distributed agency. If so, then manipulating the degree of influence participants have over their partner's behavior should boost extended self-agency when leading and extended otheragency when following. Experiment 2 confirmed these predictions. We also found synchrony created a sense of joint-agency. These results show how interpersonal synchrony can modulate a core aspect of the self.

Keywords: synchrony; self; agency; joint action; coordination

\section{Introduction}

Interpersonal synchrony - the matching of rhythmic behavior between individuals - is a common component of cultural traditions such as music making, dancing, or marching (Ehrenreich, 2006), and also occurs informally and unintentionally in day to day life during dyadic interactions (Condon \& Ogston, 1967; Shockley, Santana, \& Fowler, 2003; Van Ulzen, Lamoth, Daffertshofer, Semin, \& Beek, 2008). Interpersonal synchrony has attracted considerable attention recently due to its reported positive social effects such as greater prosocial feelings and behavior directed towards others (see reviews by Mogan, Fischer, \& Bulbulia, 2017; Rennung \& Göritz, 2016; Vicaria \& Dickens, 2016). One of the proposed theoretical mechanisms for synchrony's social effects is that it leads to a merger of the self and other (Hove, 2008; Smith, 2008; Tarr, Launay, \& Dunbar, 2014), with recent studies showing greater self-reported self-other overlap after synchrony (Fessler \& Holbrook, 2014; Reddish, Fischer, \& Bulbulia, 2013; Wiltermuth, 2012a, 2012b) and with self-other overlap mediating synchrony’s 
effects on social bonding and cooperation (Lang, Bahna, Shaver, Reddish, \& Xygalatas, 2017; Reddish et al., 2013). This suggests that interpersonal synchrony not only influences interpersonal relationships but may also influence people at a more fundamental level - the sense of self. There is some anecdotal anthropological evidence in support of this conjecture (Hanna, 1977; McNeill, 1995; Radcliffe-Brown, 1948). For example, McNeill (1995), reflecting on his experience of synchronized military drilling, describes: "a strange sense of personal enlargement; a sort of swelling out" (p.2).

However, as far as we are aware, research directly exploring if and how synchrony might affect the self has yet to be conducted. Therefore, in this paper we examined if interpersonal synchrony may influence the self. As the self is a complex construct with multiple dimensions (Baumeister, 1998), we focused on one key aspect of the self that might be particularly relevant to synchrony: the sense of agency - the feeling that we are the cause behind some action or event in the world.

The term synchrony has been used to describe a variety of coordinated interpersonal behaviours (Bernieri \& Rosenthal, 1991). The most striking and the most commonly researched in the experimental psychological literature is in-phase behavior matched synchrony (i.e., moving the same way at the same time). Current theories for how our sense of agency is generated (Pacherie, 2013; Synofzik, Vosgerau, \& Newen, 2008; Wegner \& Sparrow, 2004) suggests that such in-phase synchrony may be particularly effective at generating the feeling that one has agency over another person's actions - what we term extended self-agency (Pacherie, 2012). Firstly, when an environmental event is paired in time with an intentional action (temporal contiguity), individuals may feel as if the event was caused by them. Timing appears critical here as the longer the delay between the behavior and the event the more diminished feelings of agency over the event become (Dewey \& Carr, 2013; Sato \& Yasuda, 2005). Moreover, 
voluntary actions are perceived as occurring more closely in time with an event than involuntary actions (Haggard, Clark, \& Kalogeras, 2002). The action and the event appear to become bound in time - a process known as intentional binding (Moore \& Obhi, 2012). When an event and a voluntary behavior are paired in time repeatedly, such as two people moving rhythmically in-phase together, a particularly strong sense of agency over the event (the other person's movement) may develop.

A second possible mechanism is through visual action feedback. When people generate a motor command, a predictive model of the behavior is generated that estimates the sensory effects of the action (Frith, Blakemore, \& Wolpert, 2000). When the sensory feedback from the resulting behavior matches the prediction, this generates a sensation of agency. When the feedback does not correspond to the prediction, a decrease in agency can be felt, even when participants are viewing their own hand (Longo \& Haggard, 2009). If visual feedback from another person's actions closely matches the prediction from one's own behavior - as would be the case with in-phase synchrony - this too may generate feelings of agency over the other person's hands. Based on these two theoretical mechanisms, we might then expect a person to feel a sense of extended self-agency over another person's actions when moving synchronously in-phase with them. Consistent with this hypothesis, two studies by Kalckert and Ehrsson $(2012,2014)$ found that intentionally moving in in-phase synchrony with a rubber hand led to a greater sense of extended self-agency over that rubber hand compared to moving out of phase. However, it has yet to be seen if synchronizing with another person who has their own agency (as is the case in interpersonal synchrony) would also produce this effect. Furthermore, in Kalckert and Ehrsson's studies participants were physically connected to the rubber hand to enable it to move in time with them. In interpersonal synchrony, there is typically no physical 
connection between people whether in laboratory experiments or real-life rituals: people are not directly causing the actions of another person. Rather, synchrony is created by information about another's behavior being observed through the senses (primarily visual or auditory) and then integrated into the other person's motor output (PhillipsSilver, Aktipis, \& Bryant, 2010).

If the sense of extended self-agency over a synchronized hand is produced primarily through the two authorship indicators described above (temporal contiguity and visual action feedback) then we might expect these differences (synchronizing with a person and having no physical connection) to have minimal impact on extended selfagency over the synchronizing hand. Seeing a hand moving repeatedly the same way and at the same time as one's own arm, irrespective of whether it is real and physically attached or not, may be sufficient to induce a sense of extended self-agency over the observed arm. Alternatively, the differences between synchronizing with a real arm and a rubber arm may be important. Not directly causing the movement of the other arm or explicit knowledge that the other arm has its own agency could override any subtle authorship indicators triggered by synchrony, negating or reducing any effect of synchrony on extended self-agency.

Synchronizing with another person also introduces additional factors which may differentially affect one's sense of agency over another. In particular, synchronizing with another person can be a joint action task with performers working together to produce a desired behavioral outcome (Reddish et al., 2013). Joint coordination can be of two broad types: symmetric and asymmetric (Pacherie, 2012). Symmetric coordination is where both performers equally adjust their behavior to each other. Asymmetric coordination is when behavioral adjustments are primarily made by one individual, for example by a follower in a leader-follower relationship. The symmetry 
of the interaction and the particular role a person plays (e.g., leader or follower) may impact the effect synchrony has on agency with the more control one has over the interaction (i.e., the more one leads) the greater the sense of extended self-agency. Furthermore, recent studies (Bolt \& Loehr, 2017; Bolt, Poncelet, Schultz, \& Loehr, 2016) have found that certain joint action tasks can create a socially emergent kind of agency: joint-agency - the sense that we are acting together (Pacherie, 2013; Seemann, 2009). Synchrony produced through joint action may also produce this sense of jointagency, although it is not clear how such joint-agency may relate to any sense of extended agency over fellow performers. Although some studies have examined the sense of agency during joint actions involving two individuals (Bolt et al., 2016; Dewey, Pacherie, \& Knoblich, 2014; Obhi \& Hall, 2011; Strother, House, \& Obhi, 2010; van der Wel, 2015; van der Wel, Sebanz, \& Knoblich, 2012; Weiss, Herwig, \& Schütz-Bosbach, 2011), none have yet specifically examined synchrony. Moreover, these studies were focused on examining the sense of agency over the outcome of an action (e.g., the sense of agency over the movement of a cursor on a screen) rather than the sense of agency over another person's actions.

In the present study we examined what effect perceiving another person's hand moving in in-phase rhythmic synchrony has on a person's sense of agency. In Experiment 1 we specifically addressed the question of whether interpersonal synchrony increases the sense of extended self-agency compared to asynchronous interaction. That is, we extended Kalckert and Ehrsson's results from a rubber hand to a person. In Experiment 2, we expand upon our results from Experiment 1 by examining whether extended self-agency and extended other-agency are influenced by the symmetry of the synchronous interaction and the role the participant plays. We also examined whether interpersonal synchrony can create a sense of joint agency. 


\section{Experiment 1}

In Experiment 1 we asked participants to rhythmically move their right arm whilst observing a purported live video stream of another participant also moving their right arm. Participants, therefore, had no direct connection with the observed arm and had knowledge that the observed arm was being moved by another individual. We used a video to obtain greater control over the observed movement and to reduce any possible extraneous social cues. Participants' movements were either in-phase (synchrony) or at a different frequency (asynchrony) to the observed arm. In the in-phase synchrony condition, temporal contiguity and visual action feedback would be maximized as differences in timing are minimal and the prediction of one's own behavior and the observed movement of another's behavior would map closely onto each other. In the asynchrony condition, temporal contiguity and visual action feedback would be less involved as there is no consistent relationship in timing or with prediction and observation. Adapting similar scales to Kalckert and Ehrsson (2012), participants were then asked how much agency they felt over the other person's hand during this task (extended self-agency), and how much agency they felt the other person had over their hand (extended other-agency). Based on the above two authorship indicators and Kalckert and Ehrsson's $(2012,2014)$ results, we hypothesized that there would be a significant interaction between the two movement conditions and the two measures of agency: synchrony would lead to a greater sense of extended self-agency than in the asynchrony condition; but synchrony would not influence the sense of extended otheragency.

\section{Method}

\section{Participants.}


Participants were 137 students recruited from the National University of Singapore. Participants were recruited through an advertisement placed on a student learning website accessible to all students and were reimbursed \$5 Singapore dollars for their time. The sample size was based on how many participants we could recruit during the academic year and data collection finished when the academic year was finished. Two participants were excluded from the analyses for not following instructions (one from the synchrony condition and one from the asynchrony condition) and one participant for the video malfunctioning (asynchrony condition) resulting in 134 participants (56\% female; mean age $=21.58$, range: $18-26$ years). For both Experiment 1 and 2 ethical approval was obtained from the National University of Singapore Institutional Review Board with all participants giving informed consent.

\section{Procedure.}

The experiment was a mixed effects design with participants randomly assigned to either the synchrony or asynchrony conditions (between subjects) and all participants answering the two agency measures (within subjects).

Movement manipulation. Participants were told that the aim of the experiment was to understand how people interact over video and that they would be performing a simple task involving interacting with another participant over video.

All instructions for the synchrony manipulation were presented on the computer and the stimuli run through Open Sesame open access experimental software (Mathôt, Schreij, \& Theeuwes, 2012). Participants were asked to move their arms in time with a metronome beat whilst watching a video of a purported second participant also performing the same movement via a live video-feed. Participants were told that other participant would likewise be watching their arm movements but "may or may not hear 
the same metronome beat as you." Although participants were told the video was livestreamed, it was in fact a pre-recorded video of a female confederate. As previous research indicated that people pay less attention to others when they are moving asynchronously (Reddish et al., 2013), participants were told to look at a black fixation cross on the screen during the video to help keep attention constant. Occasionally this fixation cross changed to yellow and participants were asked to count the number of times it changed. The arm movement involved participants moving their right arm from a vertical upright position to a horizontal position resting on a desk, bending at the elbow (see Figure 1). Participants were initially shown a short video of how to do this and they were then given a short practice trial.

Participants performed the arm movements in time with eight 30 second metronome rhythms, each of a different frequency, with a short rest in between. The different rhythm frequencies were to manipulate synchrony and also helped to keep participants focused on the task and reduce boredom. In the synchrony condition, the order of the rhythms was the same between the participant and the confederate in the video. In the asynchrony condition, the confederate in the video had a different order of rhythms resulting in the participant and confederate moving at different speeds to each other. All participants across conditions heard the metronome rhythms in the same order. Four tambourine beats were played at the start of each metronome rhythm to give participants a feel for the speed of the beat. The metronome rhythm involved a low drum sound and a high tambourine sound. Participants lowered their arm so that it touched the desk on the first low drum sound, then they raised their hand so that it reached its original position on the high tambourine sound.

[Figure 1 about here] 
Questionnaire. After the synchrony manipulation, participants filled in an online questionnaire about the task. They were first asked how many times they observed the fixation cross change to yellow as a control measure of attention. Next, participants were given 8 questions adapted from Kalckert and Ehrsson (2012) which were designed to tap into participants' sense of extended self-agency and extended other-agency. These were presented one at a time on screen in random order. Participants were asked to rate how much they agreed with the 8 statements by using a sliding scale from -3 (totally disagree) to 3 (totally agree) with 0 anchored by 'uncertain'. Four items measured the sense of extended self-agency $($ Cronbach $\alpha=.83)$ :

(1) The hand on the screen moved just like I wanted it to, as if it was obeying my will;

(2) I felt as if I was controlling the movements of the hand on the screen;

(3) I felt as if I caused the movements of the hand on the screen;

(4) Whenever I moved my hand I expected the hand on the screen to move in the same way.

Four items measured the sense of extended other-agency (Cronbach $\alpha=.88$ ):

(1) I felt as if the hand on the screen was controlling my will;

(2) I felt as if the hand on the screen was controlling my movements;

(3) I felt as if the hand on the screen caused the movement in my hand;

(4) When I saw the hand on the screen move I expected my hand to move in the same way.

The mean of each scale was used in analyses. 
As a manipulation check, the same four items used by Reddish et al. (2013) to measure perceived synchrony were included, for example: "did you feel the other participants and yourself moved in unison with each other?" (Cronbach $\alpha=.85)$. The control variable of perceived attention was assessed with one item: "Did you pay attention to the other participant". These items were presented on a sliding scale from 0 (not at all) to 6 (very much so). After this questionnaire participants performed another task examining a different issue which is not the focus of the current paper and answered a second questionnaire in relation to this task.

To check that participants did not detect that the videos were pre-recorded, a question was included that asked about the timing in the video connection. Five options were given: perfect, near instantaneous, there was a short delay, there was a long delay, and the video did not seem like a live stream. This final option was the critical response to assess the believability of the videos. Analyses of this data, the manipulation check, and the control variable of attention can be found in the Supplementary Information. Finally, participants were asked demographic questions and two open ended questions about what they thought the purpose of the study was and if they had any further comments. Participants were then paid and debriefed.

\section{Results}

To examine the influence that our synchrony manipulation had on our measures of agency we conducted a mixed design ANOVA with agency direction as the within subject factor (extended self-agency, extended other-agency) and movement as the between subject factor (synchrony, asynchrony). The predicted movement by agency direction interaction was not significant, $F(1,132)=.74, p=.391, \eta_{\mathrm{p}}{ }^{2}=.01$; neither was there a main effect of agency direction, $F(1,132)=.23, p=.630, \eta_{\mathrm{p}}{ }^{2}<.01$. However, there was a main effect of condition with participants in the synchrony condition 
reporting higher scores on our agency measures $(M=-0.16, S D=1.17, n=67)$ than in the asynchrony condition $(M=-1.20, S D=1.17, n=67), F(1,132)=26.86, p<.001$, $\eta_{\mathrm{p}}^{2}=.17$. Independent samples $t$-tests confirmed that synchrony increased both extended self-agency, $t(132)=4.02, p<.001, d=0.69$, and extended other-agency, $t(132)=4.79, p<.001, d=0.83($ see Table 1 and Figure 2$)$. Both types of agency were strongly positively correlated with each other, $r(132)=.52, p<.001$.

To help in the interpretation of these results we performed some further analyses on the agency data. In the synchrony condition, the means for extended self-agency, $t(66)=-1.38, p=.172$, and extended other-agency, $t(66)=-0.42, p=.67$, were not significantly different than zero. In the asynchrony condition, the means for extended self-agency, $t(66)=-7.59, p<.001$, and extended other-agency, $t(66)=-7.37, p<.001$, were significantly lower than zero. The proportion of participants responding positively to the agency questions were significantly higher in the synchrony condition than in the asynchrony condition for both extended self-agency, $\chi^{2}(1, \mathrm{~N}=134)=6.54, p=.018$ (36\% for synchrony versus $16 \%$ for asynchrony) and extended other-agency, $\chi^{2}(1, \mathrm{~N}=$ $134)=9.67, p=.003(46 \%$ versus $21 \%)$.

[Table 1 about here]

[Figure 2 about here]

\section{Discussion}

In support of our main hypothesis, we found that moving in phase-matched synchrony with another person influenced participants' sense of extended self-agency compared to asynchrony, with participants more likely to agree with statements (or less likely to disagree) that they had some degree of agency over the other person's actions. This 
suggests that compared to asynchronous movement, synchrony does influence a person's sense of extended self-agency over another person.

However, our results diverged from Kalckert and Ehrsson's (2012, 2014) findings in two important ways. Firstly, in contrast to what we predicted and what Kalckert and Ehrsson found, synchrony also resulted in a greater sense of extended other-agency compared to asynchronous movement. One possibility for this result is that synchrony led to greater extended self-agency for some participants and greater extended other-agency for other participants. This could be because some participants may have subtly felt they led the interaction which boosted a sense of extended selfagency, whereas other participants felt that they followed which boosted extended other-agency (Wegner \& Sparrow, 2007). However, the strong positive correlation between the measures indicates that this is unlikely. Another interpretation is that it might reflect the perceived collaborative nature of interpersonal synchrony. In the synchrony condition, although the participants were not explicitly told to coordinate with the other participant and did not need to coordinate to complete the required task, they might still have interpreted the task as a joint action with both performers adjusting their behaviors to each other. (In reality this was not the case as the video was prerecorded; but as participants were led to believe that it was a live video, it may have created the illusion that coordination was symmetrical). Believing the interaction was symmetrical may have led to a sense of distributed agency: participants felt that they had some influence over the other participant's behavior (so boosting extended selfagency), but also felt that the other participant had some influence over them (so boosting extended-other agency). The greater extended other-agency in the synchrony condition may therefore reflect this sense of mutual influence. 
The second critical way our results differed from Kalckert and Ehrsson's was that our mean ratings of extended self-agency ratings were lower, with the means for both extended self-agency and extended other-agency not being significantly different than zero in the synchrony condition. Although this suggests that participants, on average, did not explicitly feel a sense of extended self-agency or extended otheragency when performing in synchrony, the large effect sizes we found do indicate that synchrony had some effect on participant's sense of agency. How then might we interpret these means? Firstly, over a third of participants did respond positively to extended self-agency questions and nearly a half of participants responded positively to extended other-agency questions, with these proportions being significantly greater than in the asynchrony condition. This suggests that during synchrony participants were more likely to perceive some agency over the other arm (and vice versa), although few participants felt in complete control of the other arm in likely recognition that the other arm was still moving at the volition of another person. Secondly, although the means were not significantly greater than zero they were not significantly below zero either, indicating that participants did not strongly disagree with statements about feeling agency over the other arm (or vice versa), as was the case in the asynchrony condition. In our study, participants' movement had no impact at all on the observed arm; participants should therefore be strongly disagreeing with any claims of agency over the observed arm. The fact that participants in the synchrony condition did not strongly reject the notion of extended self-agency suggests that synchrony still subtly influences cues about agency. The lack of physical contact with the other arm along with knowledge that the other arm had its own agency may have dampened the effect, hence producing lower means than observed in Kalckert and Ehrsson's (2012, 2014) studies, and could suggest that these factors may also be important authorship indicators for 
synchrony. Thirdly, if, as we argue, our effects are due to participants having a sense of distributed agency, a response of around zero may reflect participants' feeling that each person's behavior is the combination of both performers' agencies: the participant does not have full control over the other person's arm because the other person's agency is also involved, hence they do not strongly agree with extended self- agency statement. However, participants still have some effect on the other's agency, hence they do not strongly disagree. Likewise, with extended other-agency. In summary, we have extended previous research by providing the first evidence that synchrony does affect one's sense of agency when synchronizing with a real person. However, in contrast to a rubber hand, synchronizing with a person increased extended other-agency as well as extended self-agency and the change in extended self-agency was less with a real hand.

If the greater reported sense of extended self-agency and extended other-agency that we found during synchrony reflects a sense of distributed agency, then making the interaction more asymmetric with the participant leading the interaction should lead to a greater sense of extended self-agency and a lower sense of extended other-agency. Likewise, if a participant had to follow the movement of the other arm, this may lead to a greater sense of extended other-agency and a lower degree of extended self-agency. In symmetric coordination, the degree of extended self-agency and extended other-agency should be between leading and following as both the participant and the other arm hold some control over the interaction. To test this hypothesis, we conducted a follow-up study (Experiment 2) where we manipulated the symmetry of the synchronous interaction (symmetrical, asymmetrical) and the role participants played (leader, follower, joint contribution). As well as measuring extended self-agency and extended other-agency, we also included measures of self-agency (the degree to which 
participants feel agency over their own actions) and joint-agency to further help us understand how to interpret the effects we found in Experiment 1.

In terms of self-agency, one prediction is that there should be a negative relationship between this construct and extended other-agency: the more a person feels another has agency over their movement, the less agency that person feels over their own action. For example, if someone tries to push you over, you might likely feel your behavior is due to the agency of the pusher, not your own volition. However, greater extended self-agency may not be related to greater self-agency. Having high control over an external event (like switching on a light) might give you the sense of extended agency over that event, but it does not make you feel more in control of your own movements. It is likely people generally feel a high degree of self-agency over their movements in everyday life. Based on this, we might expect lower self-agency when extended other-agency is strong (the follower condition) due to participants relinquishing much of their control. However, participants should still retain a high amount of self-agency when they are jointly coordinating or leading the interaction.

The measure of joint-agency was included to further test whether the synchronous task in Experiment 1 was interpreted as a joint-action (even though no joint action was required or present). If so, then we would expect synchrony to generate a high sense of joint-agency. To further test this we also compared synchrony created incidentally by performers hearing the same rhythmic stimulus (as per Experiment 1) to a condition in which joint-action was explicitly primed by instructing participants they needed to cooperate during the synchrony task. If the incidental synchrony is interpreted as a joint action task even though no joint action is required (as we hypothesize), we would expect little difference in joint agency between this condition and when synchrony is explicitly primed as a joint-action. 


\section{Experiment 2}

There were four experimental conditions in Experiment 2: two symmetric conditions and two asymmetric conditions. In the two asymmetric conditions we manipulated the role participants had. In the leader condition, participants dictated the speed of movement of the joint-action. In the follower condition, participants had to follow the speed of movement of their partner. In the two symmetric conditions, participants played an equal role in creating synchrony, but we manipulated the explicit instruction to cooperate through joint action. The observation condition had similar instructions to the synchrony condition in Experiment 1 with participants passively observing the other participant while keeping in time with the metronome. In the explicit cooperation condition participants were explicitly told that cooperation was necessary to complete the task.

We hypothesized that there would be an interaction between the four experimental conditions and the direction of agency (extended self-agency and extended other-agency). Specifically, we made the following predictions:

H1: For extended self-agency, the leader condition would have the highest mean, followed by the symmetric conditions, with the follower condition having the lowest mean (leader $>$ symmetric $>$ follower). A secondary hypothesis $(\mathrm{H} 1 \mathrm{~b})$ was that the explicit cooperation condition and observation condition would not differ.

H2: For extended other-agency, the follower condition would have the highest mean, then the symmetric conditions, with the leader condition having the lowest mean (follower $>$ symmetric $>$ leader). A secondary hypothesis $(\mathrm{H} 2 \mathrm{~b})$ was that the explicit cooperation condition and observation condition would not differ.

We hypothesized that there would be a main effect of the four experimental conditions on self-agency. Specifically, we predicted that: 
H3. For self-agency, the follower condition would have the lowest mean, the leader condition would have the highest mean, and the two symmetric conditions in between as the other performer has some influence over the participant (leader > symmetric $>$ follower). A secondary hypothesis $(\mathrm{H} 3 \mathrm{~b})$ was that the explicit cooperation condition and observation condition would not differ.

We hypothesized that there would be a main effect of the four experimental conditions on joint-agency. Based on previous findings that joint-agency is greatest when coordination is symmetric (Bolt et al., 2016), we predicted that:

H4. For joint-agency: the symmetric conditions would have a higher mean than the asymmetric condition. A secondary hypothesis (H4b) was that the explicit cooperation condition and observation condition would not differ. However, we had no $a$ priori prediction for whether the two asymmetric conditions would differ.

As more exploratory research, we also included measures of self-other overlap and prosociality. This was to be able to link our dependent measures of agency with previous research that has found synchrony increases self-other overlap and prosociality. One possibility is that synchrony's increase in self-other overlap represents an expansion of both the representation of self and the other. This might map on to the greater extended self-agency and extended other-agency we found in Experiment 1. If so, then we might expect that the degree that self-agency expands over the other, combined with the degree other-agency expands over the self (i.e., the sum of extended self-agency and extended other-agency), would be positively correlated with self-other overlap. In terms of prosociality, as previous research has found self-other overlap mediates synchrony's effects on social bonding and cooperation, if the sum of extended self-agency and extended other-agency is related to self-other overlap, we should expect it to also be positively related to social bonding. 


\section{Method}

Participants.

Participants were 101 students recruited through the same method as Experiment 1 and were reimbursed \$5 Singapore dollars for their time. We aimed to collect 100 participants based on the time frame we had to complete data collection and the rate of recruitment from Experiment 1. Due to an error one extra participant was recruited. Three participants were excluded from analyses as they did not complete the questionnaire (one each from the leader, explicit cooperation, and observation conditions) leaving 98 participants that were analyzed $(58.2 \%$ female; mean age $=$ 22.58, range: $18-28$ years).

\section{Procedure.}

The experiment was a mixed effects design. Participants were randomly allocated to one of the four conditions (between-subjects), with all participants responding to the four agency scales (within-subjects).

Rather than having participants synchronize with a video, we had a confederate synchronize with the participant in the same room. This allowed for us to better model both symmetric synchrony (where both performers equally adjust their behavior to each other) and asymmetric synchrony (where the adjustments are primarily made by one individual) which we could not do with a pre-recorded video as per Experiment 1. Furthermore, intentionally following someone asynchronously was not possible in this study: moving at a different frequency to another requires actively trying not to follow their movement. This is supported by studies that show people get pulled into the rhythms of others when performing a similar behavior (Issartel, Marin, \& Cadopi, 2007; Richardson, Marsh, \& Schmidt, 2005). For this reason, and because the aim of 
Experiment 2 was to examine the effect of role and symmetry on agency, we did not manipulate synchrony.

A screen was erected between the confederate and the participant such that they could only see each other's right arm which was outstretched on a table in front of them. This set-up was to help reduce any confounding social factors but allowed the confederate and participant to observe each other's arm movements. As a further improvement from the first experiment, two different confederates were used: a male confederate for male participants and a female confederate for female participants to increase the perceived similarity of their arms.

Participants were told that the aim of the experiment was to understand how people coordinate their actions. When participants arrived at the laboratory they were seated in a cubicle with a sliding door. They were asked to read the participant information sheet while the experimenter waited for the other 'participant' outside. The experimenter then exited the laboratory and about 30 seconds later re-entered and brought the confederate to an adjacent cubicle. Because of potential sound leakage between cubicles, all instructions were repeated to the confederate as per the participant. The experimenter re-entered the participant's cubicle and asked the participant to read through the experimental instructions that were presented on the computer. This was repeated with the confederate. In the observation condition participants were given the same instruction as in Experiment 1: that the aim of the task was to move their arm intime with a metronome rhythm. In the other three conditions, participants were explicitly told that the aim of the task was "for you and the other participants to move your arms in-time with each other". In the explicit cooperation condition participants were also told that "to move in time, you and the other participant will need to work together". In the leader condition participants were informed that "only you will hear 
the metronome rhythm ... therefore, to move in time, the other participant will need to follow your movement". In the follower condition participants were told that "only the other participants will hear the metronome rhythm ... therefore, to move in time, you will need to follow the other participant's movement".

Once the participant had read through the instructions, the experimenter observed the participant practicing the arm movements to make sure the instructions were understood. After repeating this with the confederate, the experimenter then led the participant to the cubicle where the confederate was seated. The confederate and participant then completed the synchrony task together. Afterwards, the participant was led back to their original cubicle where they completed an on-line questionnaire. Participants were then thanked and paid. A delayed debriefing was emailed to participants after data completion to prevent knowledge that the other participant was a confederate being made known to future participants.

Synchrony task. Participants performed the same arm movement as in Experiment 1 with the same metronome stimulus. As previous research had found that the degree of coordination impacted joint-agency (Bolt et al., 2016) we included an objective measure of synchrony. A foam pad was positioned such that the side of the participants' and confederates' hands pressed it when it reached its horizontal nadir. This sent a trigger through a USB connection to record a time stamp of each press of the foam pad. Analysis of this data can be found in the Supplementary Information.

Questionnaire. Participants first completed the same agency questions as in Experiment 1 using the same scale, but with the phrase "the hand on the screen" replaced by "the 
other arm" (extended self-agency Cronbach $\alpha=.85$; extended other-agency Cronbach $\alpha$ $=.85)$. Three questions were designed to tap into 'self-agency' (Cronbach $\alpha=.87)$ :

(1) My arm moved just like I wanted it to, it was obeying my will.

(2) I felt as if I was in full control of the movements of my arm.

(3) I felt as if I caused the movements of my arm.

In terms of joint-agency, Bolt et al.(2016) is the only other study thus far to use an explicit measure for this construct. However, they measured joint-agency on a single dimension ranging from independent control to shared control. As we wanted to measure joint-agency as a separate construct from self-agency we created our own measure which also mirrored the items asked for our other agency measures (Cronbach $\alpha=.78)$ :

(1) Our arms moved just like we wanted them to, they were obeying our will.

(2) I felt as if we were controlling the movements of our arms.

(3) I felt as if we caused the movements of our arms.

(4) I felt like my arm and the other arm had become 'our' arms.

The mean of each agency scale was used in analyses.

To measure self-other overlap we used the Dynamic Identity Fusion Index (DIFI; Jiménez et al., 2016), which involves participants moving a circle representing their self to overlap with another circle representing the other participant. Participants were asked to move the circle "to the position that best captures your relationship with the other participant". This produced two measures: the degree to which the circles overlap, and the distance between the centers of the circles. However, as we were specifically interested in overlap and as previous research has found the overlap metric 
was a better indicator of self-other fusion (Jiménez et al., 2016), we only examined this measure.

We included two measures of social bonding - attraction and entitativity. Attraction was measured with two items on sliding scales from 0 (not at all) to 6 (very much so): "Would you like to meet the other participant?" and "Do you think the other participant is friendly?". For entitativity, we used the 5 item scale as used by Reddish, Tong, Jong, Lanman, and Whitehouse (2016), (e.g., "Did you feel you were on the same team with the other participant?") with the same sliding scale as for attraction. As these two constructs were strongly correlated, $r(96)=.43, p<.001$, we averaged them together into a combined social bonding scale (Cronbach $\alpha=.87)$.

As a manipulation check we asked participants: "Do you feel you led the movement of the other participant?" and "Do you feel you followed the movement of the other participant?" on a sliding scale from 0 (not at all) to 6 (very much so). We included a number of control variables using this same sliding scale to check the conditions did not vary in other unexpected ways: cooperation ("Did you feel you and the other participant cooperated?'); prediction ('Were you able to predict the other participant's movements?"); and attention (“Did you pay attention to the other participant's movements?"). Other control variables we included were: participants' perceived success of their own performance ("How successful do you feel you were at your task?"), their partner's performance (“How successful do you feel the other participant was at their task?"), and the joint performance ("How successfully do you feel you and the other participant worked together?" ), asked on a sliding scale from 0 (not successful at all) to 6 (very successful); if the movement task was enjoyable and if it was difficult using a scale from -3(totally disagree) to 3(totally agree); participants' preference "for one hand over another on common tasks such as writing, holding a 
spoon, and throwing" from -10 (left dominant) to 10 (right dominant); participants' mood assessed with the 5-point self-assessment manikin (Bradley \& Lang, 1994) with a sliding scale to measure both valence ( -2 to 2 ) and arousal ( 0 to 4$)$; and perceived synchrony assessed with the same measure as in experiment 1 (Cronbach $\alpha=.79)$. Finally, participants were asked demographic questions and two open ended questions about what they thought the purpose of the study was and if they had any further comments.

As the manipulation check and control variables were not a key focus for this study, results for these variables are reported in the Supplementary Information.

\section{Results}

To examine the effect of our manipulation on extended self-agency and extended otheragency we ran a mixed design ANOVA with agency direction (extended self-agency, extended other-agency) as the within subject factor and the four experimental conditions as the between subject factor ${ }^{1}$. We found a main effect of agency direction, $F(1,94)=$ $14.94, p<.001, \eta_{\mathrm{p}}^{2}=.14$, with extended self-agency $(M=0.12, S D=1.47)$ higher than extended other-agency $(M=-0.46, S D=1.45)$. However, this was qualified by a significant interaction with experimental condition, $F(3,94)=20.39, p<.001, \eta_{\mathrm{p}}{ }^{2}=.39$.

[Table 2 about here].

To examine the simple effects of this interaction, we compared conditions for extended self-agency and extended other-agency separately using contrast analysis with Bonferroni corrections (see Table 2 for descriptive statistics). Initially we compared the leader versus the follower condition, then we compared each of these conditions to the combined symmetric conditions, and lastly, we compared the two symmetric conditions. 
As can be seen in Figure 3, extended self-agency was higher in the leader condition compared to the follower condition $(p<.001, d=1.32)$ and the symmetric conditions $(p$ $=.048, d=0.65)$, and the follower condition was marginally lower than the symmetric conditions $(p=.052, d=0.63)$. For extended other-agency, the follower condition was higher than the leader condition $(p<.001, d=1.25)$ and the symmetric conditions $(p<$ $.001, d=0.96)$, but the leader condition did not differ from the symmetric conditions ( $p$ $=.610, d=0.36)$. The observation condition did not differ from the explicit cooperation condition for both extended self-agency $(p=1.00, d=0.13)$ and extended other-agency $(p=1.00, d=0.15)$. Extended self-agency was significantly greater than zero in the leader condition, $t(23)=3.75, p=.001$, and extended other-agency was significantly greater than zero in the follower condition, $t(25)=2.14, p=.042$.

A one-way ANOVA revealed a main effect of experimental condition on selfagency, $F(3,94)=9.10, p<.001, \eta_{\mathrm{p}}^{2}=.23$. Performing the same contrast analyses as above, we found that self-agency scores were lower in the follower condition than in the leader condition $(p<.001, d=1.19)$ and the symmetric conditions $(p<.001, d=0.96)$; but the leader condition did not differ from the symmetric conditions ( $p=1.00, d=$ $0.07)$ nor did the symmetric conditions differ $(p=1.00, d=0.30)$. Self-agency was significantly greater than zero for all condition except for the follower condition: $t(24)=$ $10.04, p<.001$ (observation); $t(22)=6.16, p<.001($ explicit cooperation $) ; t(23)=$ $12.92, p<.001$ (leader); $t(25)=1.44, p=.162$ (follower).

[Figure 3 about here]

A one-way ANOVA found no significant main effect of condition on jointagency, $F(3,94)=0.26, p=.856, \eta_{\mathrm{p}}^{2}=.01$. Joint agency was significantly greater than 
zero across all four conditions: $t(24)=3.36, p=.003$ (observation); $t(22)=4.25, p<$. $001($ explicit cooperation); $t(23)=3.69, p=.001$ (leader); $t(25)=2.01, p=.046$ (follower). As we specifically predicted a difference in joint agency between the symmetric conditions and the asymmetric conditions we conducted a $t$-test on this pairwise comparison, but found no effect, $t(96)=0.36, p=.720, d=0.07$. Notably, joint-agency did correlate with perceived joint success, $r(96)=.54, p<.001$ and perceived synchrony, $r(96)=.59, p<.001$, but not with our objective measure of synchrony, $r(64)=-.04, p=.728$. Correlations between all four agency measures can be found in the Supplementary Information.

As predicted, there was a significant positive relationship between the sum of extended self-agency and extended other agency and our measures of self-other overlap, $r(96)=.38, p<.001$, and social bonding, $r(96)=.41, p<.001$. Self-other overlap and social bonding were also strongly positively related, $r(96)=.48, p<.001$.

\section{Discussion}

Our key novel finding from Experiment 2 was that the symmetry of the interaction and the role the participant played in creating synchrony are also important factors in influencing participants' sense of agency. As predicted, the leader condition resulted in higher extended self-agency than the follower condition and the symmetric conditions (H1). Also, as predicted, the follower condition had the highest mean extended otheragency $(\mathrm{H} 2)$. Together, these results support our interpretation of Experiment 1 that the greater extended self-agency and extended other-agency observed in the synchrony condition was expressive of a perceived distribution of agency due to the task being interpreted as a joint action. The more one influences the other participant's movements, as in the leader condition, the greater one feels agency over them; conversely the more one is influenced by the other person, as in the follower condition, 
the more one feels the other has agency over them. When participants both contribute to the synchrony as in the symmetric conditions, agency is more equally distributed across the actors.

Mean extended self-agency was significantly greater than zero in the leader condition. Therefore, this is the first data we know of to show that interpersonal synchrony can create a strong explicit sense of extended self-agency. However, one must be leading the interaction. Likewise, the novel finding that mean extended otheragency was significantly greater than zero in the follower condition shows that interpersonal synchrony can also create a strong explicit sense of extended otheragency.

Mean joint-agency was positive and significantly greater than zero across all conditions indicating that synchrony did create a sense of joint-agency. The finding that the observation condition created a sense of joint-agency and that the explicit cooperation and observation conditions did not significantly differ across any of our agency measures (H1b, H2b, H3b, H4b) suggests that even when joint-action is not explicitly required to create synchrony, participants may still be regarding the task as a collaborative one. This is further evidence in support of our proposal that the greater extended self-agency and extended other-agency observed in the synchrony condition in Experiment 1 was due to the synchrony being perceived as collaborative.

Interestingly, joint-agency did not differ significantly across any of the four conditions (H4). This is in contrast to extended self-agency and extended other-agency which were affected by role and symmetry, suggesting joint-agency is not simply a product of extended self-agency and extended other-agency. Instead, as also found by Bolt et al. (2016) and theorized by Pacherie (2012), joint-agency appears more reflective of the perceived success of the cooperative task - as shown by the strong 
positive correlations between joint agency and perceived joint success and perceived synchrony. If this is a general property of joint agency across joint action tasks and not just restricted to synchrony, an implication is individuals who may have little impact on a group's performance may still feel a strong sense of joint agency if the group is successful. For example, a supporter of a sports team should feel a greater sense of joint-agency with the actual athletes after a victory as opposed to a loss, even though their actual impact on the result was negligible.

The reduction of self-agency in the follower condition (H3) implies that when participants followed another in a synchronous action, they felt less in control over their own actions. Although previous research has found that agency can be extended over other objects or people (e.g., Pronin et al., 2006; Wegner et al., 2004), this is the first result we know of where a simple synchronous interaction has resulted in a reduction of one's sense of self-agency.

The leader condition did not have significantly different self-agency than the symmetric conditions. As all three of these conditions had high levels of agreement for the self-agency questions with means significantly greater than zero, this indicates that participants retained a high level of agency over their own movement when contributing equally to the joint action (as in the symmetric conditions) or leading. It also suggests that being a leader does not boost the sense of self-agency above symmetric jointaction. Our data suggest that it is only possible to reduce the sense of self-agency when one follows a partner.

Self-other overlap was positively correlated with the sum of extended selfagency and extended other-agency. This is consistent with our idea that synchrony's boost in self-reported self-other overlap as found in previous research may, in part, be generated by the expansion of one's sense of self-agency and/or one's sense of other- 
agency. Both measures were also strongly positively correlated with our social bonding measure. This could suggest that one possible mechanism through which synchrony boosts prosociality is by influencing one's sense of agency, which in turn generates a feeling of self-other overlap, which leads one to feel 'as one' with their fellow performer and hence more likely to help them. However, as this analysis is correlational future experimental research is needed to examine if manipulating agency does cause greater self-other overlap and prosociality.

One potential critique of our findings is the effects may be a product of demand characteristics. As some participants were instructed to lead or follow, they may have responded to the agency questions in ways that they thought leaders and followers should respond rather than due to the manipulation per se. There are a few reasons why this is unlikely to be the case. Firstly, none of the key words that are used in the agency measures such as 'will', 'caused', 'control' were used in the instructions. Likewise, there is no mention of leading or following in the dependent variable. Therefore, there is no simple relationship between the instructions and the agency dependent variable, reducing the likelihood of a simple priming by the instructions. Secondly, when participants were asked in the questionnaire what the purpose of the study was, only eight participants mentioned anything about leading or following (4 in the leader condition, 2 in the follower condition). Of these, only one participant mentioned anything about what it "feels like" to lead or follow. The rest of these participants focused on the actual process of synchronizing or the social effects (e.g., "if people will follow one another when doing a task"). This would suggest that leading or following was not very salient in participants' minds nor did participants explicitly link it with agency. Thirdly, participants in the explicit cooperation conditions were told that the aim of the task was for them to "work together". If the instructions were priming 
participants to respond to agency questions in a certain way rather than the experience of the interaction, then we might expect this explicit instruction to also be affected by demand characteristics, thus boosting joint agency. However, joint agency was not significantly different across the conditions.

\section{General discussion}

In this paper we explored how interpersonal synchrony influences a person's sense of agency. We originally predicted that moving in phase-matched synchrony with another person would heighten the sense of agency over that person's behavior. In Experiment 1 we found data consistent with this hypothesis, but we also found evidence of a heightened sense that the other person has agency over the participant's behavior compared to asynchronous movement. We hypothesized that this may have been due to the movement task being perceived as a joint one, and hence participants felt that agency was distributed across both individuals. Experiment 2 provided evidence in support of this conjecture by manipulating the role and symmetry of the synchronous interaction. If a participant felt they had some influence over the timing of another's actions - whether by leading the synchronous interaction or through mutual influence this boosted the feeling that they had agency over the other person. Likewise, if a participant felt the other person had some influence over the timing of their actions whether this was because they followed or were mutually influenced by the other person - this boosted the feeling that the other person had agency over their actions, and when following, led to a reduction of their sense of self.

The increase in extended self-agency in Experiment 1 suggests that the authorship indicators of temporal contiguity between an action and an event and visual action feedback may play a role in influencing the sense of agency in synchronous tasks when compared to asynchronous tasks. This could imply that in-phase behavior 
matched synchrony would lead to a greater change in one's sense of agency than other types of coordinated behavior that is not closely matched in form and timing. However, the differences between our data and Kalckert and Ehrsson's data suggests that knowledge that the object one is coordinated with has its own agency and the presence or absence of direct physical contact with this object are also important agency cues. In Experiment 2, the outcome of the participants' interaction was the same across conditions: participants moved in-time, the same number of times, and at the same speed as the confederate. The finding that the role the participant played modulated participants' sense of agency suggests that how synchrony is created and the particular coordination dynamics are also important agency cues. Therefore, the degree that any particular type of interpersonal coordination may impact one's sense of agency will likely be dependent on these factors and possibly other agency indicators (see Wegner \& Sparrow, 2004) meaning whether synchrony influences one's sense of agency more than other types of interpersonal coordination remains an open empirical question.

A further consideration is that the authorship indicators of temporal contiguity and visual action feedback may exert a greater influence on the implicit, nonconceptual, feeling of agency; whereas higher order cognitive factors such as knowledge that the co-actor has his or her own agency and the perceived role in the interaction may have more of an impact on explicit judgements of agency as employed in our study (Synofzik et al., 2008; Weiss, Tsakiris, Haggard, \& Schütz-Bosbach, 2014). Future studies could use implicit measures of agency such as sensory attenuation (Weiss et al., 2011) or the intentional binding effect (Haggard et al., 2002) to examine synchrony's effect on one's feeling of agency. Notably implicit and explicit measures of agency appear to tap into different aspects of agency and hence may produce divergent 
results from what we obtained here (Dewey \& Knoblich, 2014; Moore, Middleton, Haggard, \& Fletcher, 2012).

The modulation of agency with the role a performer plays in a synchronous performance may have some interesting implications outside of the laboratory. For instance, in partner dancing, there is often a 'lead' and a 'follow' synchronously working together. After such a dance the lead may feel greater agency over the follow, with the follow even feeling a decreased sense of self-agency. Another relevant area could be with religious rituals. Rhythmic synchrony is common during religious rituals, and following others or an external beat during such performances (which based on our results may lower one's sense of self-agency) may help facilitate ideas that external powers (such as spirits or gods) may be controlling one's movements. As an example, Whitehouse (1996) describes a ritual where teams of males synchronously move large sections of bamboo, passively following where the group movement leads them. The dramatic movements of the bamboo are in turn attributed to external spirits. The impact of role on agency may also be important to investigate in synchronous military marching. Previous research has found that synchrony decreases the perceived formidability of enemies (Fessler \& Holbrook, 2014; cf., Hagen \& Bryant, 2003). This could be due to an expanded sense of self-agency manifested during symmetric collective marching that leads individuals to feel they are more powerful and hence have more confidence and less fear going into difficult situations such as warfare. However, if cadets perceive themselves as following the marching orders of an officer, our results above suggest that this may in fact lead to a reduced sense of self-agency and, in turn, a decreased sense of power. Future research examining cadets' sense of agency, power, and perceived role may help to clarify this. 
In the two studies reported here we varied multiple aspects of synchronous interactions: relative timing, symmetry, role, explicit instructions to cooperate, and videotaped versus in-person interaction. Nevertheless, there are other aspects of synchronous interactions as seen in the real world that may impact the effect it has on individuals' sense of agency (see Pacherie, 2012). One potentially important dimension is group size (Mogan et al., 2017). We only examined dyadic interactions. Yet synchronous performances can grow to tens of thousands (and potentially many more) as commonly seen in sports stadiums around the world. In large group synchronous performances an individual may indeed lose their sense of self in the collective rather than feel an extended sense of agency over the whole group, potentially through an increase in the salience of the group's identity (Turner, Oakes, Haslam, \& McGarty, 1994). However, recent research has shown that individuals can also fuse with a group (identity fusion) such that their personal self becomes functionally equivalent to their group identity (Swann, Gomez, Seyle, Morales, \& Huici, 2009). Such fused individuals appear to retain a strong sense of personal agency in group contexts (Swann \& Buhrmester, 2015). This could suggest that when such fused individuals perform synchronously with their group they may still feel a sense of extended agency over the group.

In conclusion, in this paper we provide the first direct evidence we know of that that simple synchronizing with another person can modify a fundamental aspect of the self - one's explicit sense of agency. However, how synchrony influences one's sense of agency appears to depend in part on how the synchrony is created, the role the performer plays in the synchronous interaction, the interaction symmetry, and in the case of the sense of joint-action, the perceived success of the synchronous interaction. This means synchrony can potentially lead to a sense of extended self-agency over 
another, a sense of extended other-agency over the self, a reduction in self-agency, and a sense of joint-agency depending on the dynamics of the interaction and how it is interpreted by performers. 


\section{Acknowledgements}

Thanks to Ai Jia Gwee, Brenda Low, Chew Yun Ping, Elissa Lim, Goh Chun Aik, and Luan Ling for help with the experiments.

This research was supported by a Large Grant from the UK's Economic and Social Research Council (REF RES-060-25-0085) entitled "Ritual, Community, and Conflict", an award from the John Templeton Foundation entitled "Religion's Impact on Human Life", an Advanced Grant from the European Research Council (ERC) under the European Union's Horizon 2020 Research and Innovation Programme (grant agreement No. 694986), an award from the Templeton World Charity Foundation entitled "Cognitive and Cultural Foundations of Religion and Morality", and Singapore Ministry of Education Academic Research Fund R-581-000-207-115.

\section{Declaration of interest statement}

There are no known conflicts of interest. 


\section{References}

Baumeister, R. F. (1998). The self. In D. Gilbert, S. T. Fiske, \& G. Lindzey (Eds.), The Handbook of Social Psychology (Vol. 1, pp. 680-740). New York: McGraw-Hill.

Bernieri, F. J., \& Rosenthal, R. (1991). Interpersonal coordination: Behavior matching and interactional synchrony. In R. S. Feldman \& B. Rime (Eds.), Fundamentals of nonverbal behavior: Studies in emotion \& social interaction (pp. 401-432). New York: Cambridge University Press.

Bolt, N. K., \& Loehr, J. D. (2017). The predictability of a partner's actions modulates the sense of joint agency. Cognition, 161, 60-65. https://doi.org/10.1016/j.cognition.2017.01.004

Bolt, N. K., Poncelet, E. M., Schultz, B. G., \& Loehr, J. D. (2016). Mutual coordination strengthens the sense of joint agency in cooperative joint action. Consciousness and Cognition, 46, 173-187. https://doi.org/10.1016/j.concog.2016.10.001

Bradley, M., \& Lang, P. J. (1994). Measuring emotion: The self-assessment manikin and the semantic differential. Journal of Behavior Therapy and Experimental Psychiatry, 25(I), 49-59. https://doi.org/10.1016/0005-7916(94)90063-9

Condon, W. S., \& Ogston, W. D. (1967). A segmentation of behavior. Journal of Psychiatric Research, 5, 221-235.

Dewey, J. A., \& Carr, T. H. (2013). When dyads act in parallel, a sense of agency for the auditory consequences depends on the order of the actions. Consciousness and Cognition, 22(1), 155-166. https://doi.org/10.1016/j.concog.2012.12.004

Dewey, J. A., \& Knoblich, G. (2014). Do implicit and explicit measures of the sense of agency measure the same thing? PLoS ONE, 9(10), e110118. https://doi.org/10.1371/journal.pone.0110118

Dewey, J. A., Pacherie, E., \& Knoblich, G. (2014). The phenomenology of controlling a moving object with another person. Cognition, 132(3), 383-397. 
https://doi.org/10.1016/j.cognition.2014.05.002

Ehrenreich, B. (2006). Dancing in the streets: A history of collective joy. New York: Metropolitan.

Fessler, D. M. T., \& Holbrook, C. (2014). Marching into battle: synchronized walking diminishes the conceptualized formidability of an antagonist in men. Biology Letters, 10(8), 20140592. https://doi.org/10.1098/rsbl.2014.0592

Frith, C. D., Blakemore, S. J., \& Wolpert, D. M. (2000). Abnormalities in the awareness and control of action. Philosophical Transactions of the Royal Society of London. Series B, Biological Sciences, 355(1404), 1771-1788.

https://doi.org/10.1098/rstb.2000.0734

Hagen, E. H., \& Bryant, G. A. (2003). Music and dance as a coalition signaling system. Human Nature, 14(1), 21-51.

Haggard, P., Clark, S., \& Kalogeras, J. (2002). Voluntary action and conscious awareness. Nature Neuroscience, 5(4), 382-385. https://doi.org/doi:10.1038/nn827

Hanna, J. L. (1977). African dance and the warrior tradition. Journal of Asian and African Studies, 12, 111-133.

Hove, M. J. (2008). Shared circuits, shared time, and interpersonal synchrony. Behavioral and Brain Sciences, 31(1), 29-30. https://doi.org/10.1017/s0140525x07003202

Issartel, J., Marin, L., \& Cadopi, M. (2007). Unintended interpersonal co-ordination: “can we march to the beat of our own drum?". Neuroscience Letters, 411(3), 174179. https://doi.org/10.1016/j.neulet.2006.09.086

Jiménez, J., Gomez, A., Buhrmester, M. D., Vazquez, A., Whitehouse, H., \& Swann, W. B. (2016). The dynamic identity fusion index: A new continuous measure of identity fusion for web-based questionnaires. Social Science Computer Review, 
34(2), 215-228. https://doi.org/10.1177/0894439314566178

Kalckert, A., \& Ehrsson, H. H. (2012). Moving a rubber hand that feels like your own: A dissociation of ownership and agency. Frontiers in Human Neuroscience, 6, 114. https://doi.org/10.3389/fnhum.2012.00040

Kalckert, A., \& Ehrsson, H. H. (2014). The moving rubber hand illusion revisited: comparing movements and visuotactile stimulation to induce illusory ownership. Consciousness and Cognition, 26, 117-132. https://doi.org/10.1016/j.concog.2014.02.003

Lang, M., Bahna, V., Shaver, J. H., Reddish, P., \& Xygalatas, D. (2017). Sync to link: Endorphin-mediated synchrony effects on cooperation. Biological Psychology, 127, 191-197. https://doi.org/10.1016/j.biopsycho.2017.06.001

Longo, M., \& Haggard, P. (2009). Sense of agency primes manual motor responses. Perception, 38, 69-78. https://doi.org/10.1068/p6045

Mathôt, S., Schreij, D., \& Theeuwes, J. (2012). OpenSesame: An open-source, graphical experiment builder for the social sciences. Behavior Research Methods, 44(2), 314-324. https://doi.org/10.3758/s13428-011-0168-7

McNeill, W. H. (1995). Keeping together in time: Dance and drill in human history. Cambridge, MA: Harvard University Press.

Mogan, R., Fischer, R., \& Bulbulia, J. A. (2017). To be in synchrony or not? A metaanalysis of synchrony's effects on behavior, perception, cognition and affect. Journal of Experimental Social Psychology, 72, 13-20. https://doi.org/10.1016/j.jesp.2017.03.009

Moore, J. W., Middleton, D., Haggard, P., \& Fletcher, P. C. (2012). Exploring implicit and explicit aspects of sense of agency. Consciousness and Cognition, 21(4), 1748-1753. https://doi.org/10.1016/j.concog.2012.10.005 
Moore, J. W., \& Obhi, S. S. (2012). Intentional binding and the sense of agency: a review. Consciousness and Cognition, 21(1), 546-561. https://doi.org/10.1016/j.concog.2011.12.002

Obhi, S. S., \& Hall, P. (2011). Sense of agency and intentional binding in joint action. Experimental Brain Research, 211, 655-662. https://doi.org/10.1007/s00221-011$2675-2$

Pacherie, E. (2012). The phenomenology of joint action: Self-agency vs. joint-agency. In A. Seemann (Ed.), Joint Attention: New Developments (pp. 343-389). MIT Press. https://doi.org/10.1007/s13164-011-0052-5

Pacherie, E. (2013). How does it feel to act together? Phenomenology and the Cognitive Sciences, 13(1), 25-46. https://doi.org/10.1007/s11097-013-9329-8

Phillips-Silver, J., Aktipis, C. A., \& Bryant, G. A. (2010). The ecology of entrainment: Foundations of coordinated rhythmic movement. Music Perception, 28(1), 3-14. https://doi.org/10.1525/mp.2010.28.1.3

Pronin, E., Wegner, D. M., McCarthy, K., \& Rodriguez, S. (2006). Everyday magical powers: the role of apparent mental causation in the overestimation of personal influence. Journal of Personality and Social Psychology, 91(2), 218-231. https://doi.org/10.1037/0022-3514.91.2.218

Radcliffe-Brown, A. R. (1948). The Andaman Islanders. Glencoe: The Free Press.

Reddish, P., Fischer, R., \& Bulbulia, J. (2013). Let's dance together : Synchrony, shared intentionality and cooperation. PloS One, 8(8). https://doi.org/10.1371/journal.pone.0071182

Reddish, P., Tong, E. M. W., Jong, J., Lanman, J. A., \& Whitehouse, H. (2016). Collective synchrony increases prosociality towards non-performers and outgroup members. British Journal of Social Psychology, 55(4), 722-738. 
https://doi.org/10.1111/bjso.12165

Rennung, M., \& Göritz, A. S. (2016). Prosocial consequences of interpersonal synchrony: A Meta-Analysis. Zeitschrift Fur Psychologie / Journal of Psychology, 224(3), 168-189. https://doi.org/10.1027/2151-2604/a000252

Richardson, M. J., Marsh, K. L., \& Schmidt, R. C. (2005). Effects of visual and verbal interaction on unintentional interpersonal coordination. Journal of Experimental Psychology-Human Perception and Performance, 31(1), 62-79. https://doi.org/10.1037/0096-1523.31.1.62

Sato, A., \& Yasuda, A. (2005). Illusion of sense of self-agency: Discrepancy between the predicted and actual sensory consequences of actions modulates the sense of self-agency, but not the sense of self-ownership. Cognition, 94(3), 241-255. https://doi.org/10.1016/j.cognition.2004.04.003

Seemann, A. (2009). Joint agency: Intersubjectivity, sense of control, and the feeling of trust. Inquiry, 52(5), 500-515. https://doi.org/10.1080/00201740903302634

Shockley, K., Santana, M. V., \& Fowler, C. A. (2003). Mutual interpersonal postural constraints are involved in cooperative conversation. Journal of Experimental Psychology-Human Perception and Performance, 29(2), 326-332. https://doi.org/10.1037/0096-1523.29.2.326

Smith, E. R. (2008). An embodied account of self-other "overlap" and its effects. In G. R. Semin \& E. R. Smith (Eds.), Embodied grounding: Social, cognitive, affective, and neuroscientific approaches. (pp. 148-159). New York: Cambridge University Press.

Strother, L., House, K. A., \& Obhi, S. S. (2010). Subjective agency and awareness of shared actions. Consciousness and Cognition, 19(1), 12-20. https://doi.org/10.1016/j.concog.2009.12.007 
Swann, W. B., \& Buhrmester, M. D. (2015). Identity fusion. Current Directions in Psychological Science, 24(1), 52-57. https://doi.org/10.1177/0963721414551363

Swann, W. B., Gomez, A., Seyle, D. C., Morales, J. F., \& Huici, C. (2009). Identity fusion: The interplay of personal and social identities in extreme group behavior. Journal of Personality and Social Psychology, 96(5), 995-1011. https://doi.org/10.1037/a0013668

Synofzik, M., Vosgerau, G., \& Newen, A. (2008). Beyond the comparator model: A multifactorial two-step account of agency. Consciousness and Cognition, 17(1), 219-239. https://doi.org/10.1016/j.concog.2007.03.010

Tarr, B., Launay, J., \& Dunbar, R. I. M. (2014). Music and social bonding: "Self-other" merging and neurohormonal mechanisms. Frontiers in Psychology, 5, 1-10. https://doi.org/10.3389/fpsyg.2014.01096

Turner, J. C., Oakes, P. J., Haslam, S. A., \& McGarty, C. (1994). Self and collective: Cognition and social context. Personality and Social Psychology Bulletin, 20(5), $454-463$.

van der Wel, R. P. R. D. (2015). Me and we: Metacognition and performance evaluation of joint actions. Cognition, 140, 49-59. https://doi.org/10.1016/j.cognition.2015.03.011

van der Wel, R. P. R. D., Sebanz, N., \& Knoblich, G. (2012). The sense of agency during skill learning in individuals and dyads. Consciousness and Cognition, 21(3), 1267-1279. https://doi.org/10.1016/j.concog.2012.04.001

Van Ulzen, N. R., Lamoth, C. J. C., Daffertshofer, A., Semin, G. R., \& Beek, P. J. (2008). Characteristics of instructed and uninstructed interpersonal coordination while walking side-by-side. Neuroscience Letters, 432(2), 88-93. https://doi.org/10.1016/j.neulet.2007.11.070 
Vicaria, I. M., \& Dickens, L. (2016). Meta-analyses of the intra- and interpersonal outcomes of interpersonal coordination. Journal of Nonverbal Behavior, 40(4), 335-361. https://doi.org/10.1007/s10919-016-0238-8

Wegner, D. M., \& Sparrow, B. (2004). Authorship processing. In M. S. Gazzaniga (Ed.), The Cognitive Neurosciences (3rd ed., pp. 1201-1209). Cambridge, MA: MIT Press.

Wegner, D. M., \& Sparrow, B. (2007). The puzzle of coaction. In D. Ross, D. Spurrett, K. H, \& S. L (Eds.), Distributed Cognition and the Will (pp. 17-37). Cambridge, MA: MIT Press.

Wegner, D. M., Sparrow, B., \& Winerman, L. (2004). Vicarious agency: Experiencing control over the movements of others. Journal of Personality and Social Psychology, 86(6), 838-848. https://doi.org/10.1037/0022-3514.86.6.838

Weiss, C., Herwig, A., \& Schütz-Bosbach, S. (2011). The self in social interactions: sensory attenuation of auditory action effects is stronger in interactions with others. PloS One, 6(7), e22723. https://doi.org/10.1371/journal.pone.0022723

Weiss, C., Tsakiris, M., Haggard, P., \& Schütz-Bosbach, S. (2014). Agency in the sensorimotor system and its relation to explicit action awareness. Neuropsychologia, 52, 82-92. https://doi.org/10.1016/j.neuropsychologia.2013.09.034

Whitehouse, H. (1996). Apparitions, orations, and rings: Experience of spirits in Dadul. In A. Howard \& J. Mageo (Eds.), Spirits in Culture, History, and Mind (pp. 173193). London: Routledge.

Wiltermuth, S. S. (2012a). Synchronous activity boosts compliance with requests to aggress. Journal of Experimental Social Psychology, 48(1), 453-456. https://doi.org/10.1016/j.jesp.2011.10.007 
Wiltermuth, S. S. (2012b). Synchrony and destructive obedience. Social Influence, 3741. https://doi.org/10.1080/15534510.2012.658653 
Footnotes

${ }^{1}$ We repeated all analyses with sex as an additional factor to check that the fact we varied the confederate with the sex of the participant did not affect our results. The only significant interaction with sex we found was a marginally significant agency direction by sex interaction, $F(1,90)=3.43, p=.067, \eta_{\mathrm{p}}^{2}=.037$ for this ANOVA; however, the more critical three-way interaction of agency direction by sex by condition was not significant, $F(3,90)=0.48, p=.700, \eta_{\mathrm{p}}^{2}=.016$. 
Table 1.

Summary statistics for Experiment 1.

\begin{tabular}{lcc}
\hline & \multicolumn{2}{c}{ Condition } \\
\cline { 2 - 3 } & Synchrony & Asynchrony \\
\hline $\mathrm{n}$ & $-0.24(1.43)$ & $-1.18(1.27)$ \\
Extended self-agency & $-0.07(1.42)$ & $-1.23(1.36)$ \\
Extended other-agency & & \\
\hline Note. Reported is the mean with standard deviation in parentheses.
\end{tabular}

Table 2.

Summary statistics of key variables for Experiment 2.

\section{Condition}

\begin{tabular}{ccccc}
\hline Leader & Follower & $\begin{array}{c}\text { Explicit } \\
\text { Cooperation }\end{array}$ & Observation & Symmetric \\
& & & & \\
\hline 24 & 26 & 23 & 25 & 48
\end{tabular}

Extended self-

$$
1.00(1.31) \quad-0.70(1.26) \quad 0.23(1.53) \quad 0.04(1.32) \quad 0.13(1.41)
$$

agency

Extended other-

$\begin{array}{llllll} & -1.16(1.37) & 0.62(1.46) & -0.60(1.28) & -0.78(1.05) & -.069(1.16) \\ \text { agency } & & & & & \\ \text { Self-agency } & 1.80(0.68) & 0.43(1.51) & 1.57(1.22) & 1.90(0.94) & 1.74(1.09) \\ \text { Joint-agency } & 0.85(1.12) & 0.64(1.56) & 0.93(1.05) & 0.73(1.09) & .83(1.07)\end{array}$

Note. Reported is the mean with standard deviation in parentheses. 'Symmetric' is the combined observation and explicit cooperation conditions. 


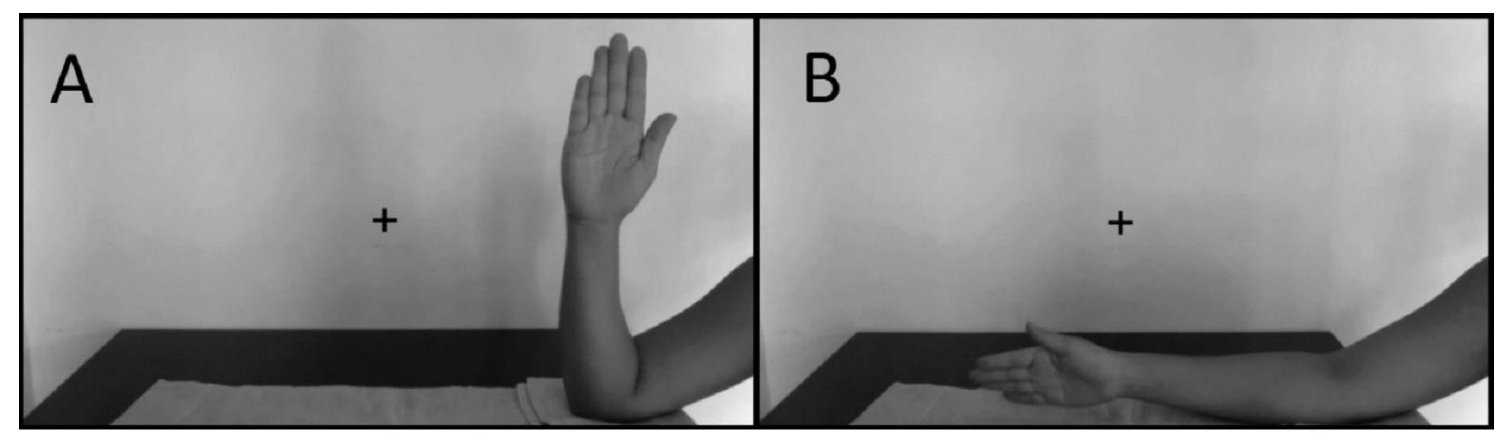

Figure 1. Video stills of the stimuli used in the movement manipulation for Experiment 1. 'A' shows the arm at the start of the movement in a vertical position. 'B' is the arm in a horizontal position.

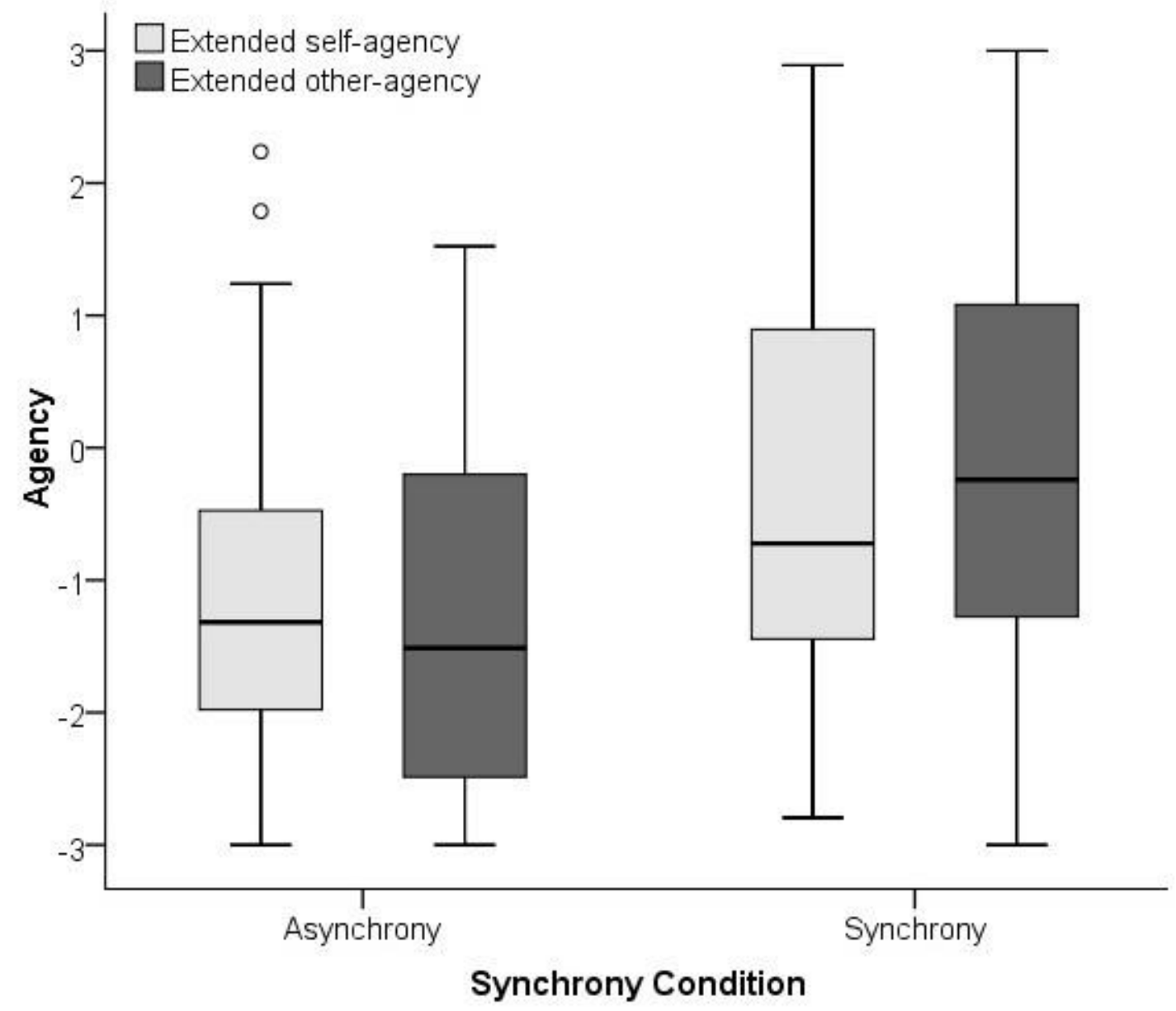

Figure 2. Boxplot of extended self-agency and extended other-agency scores by movement condition for Experiment 1. 


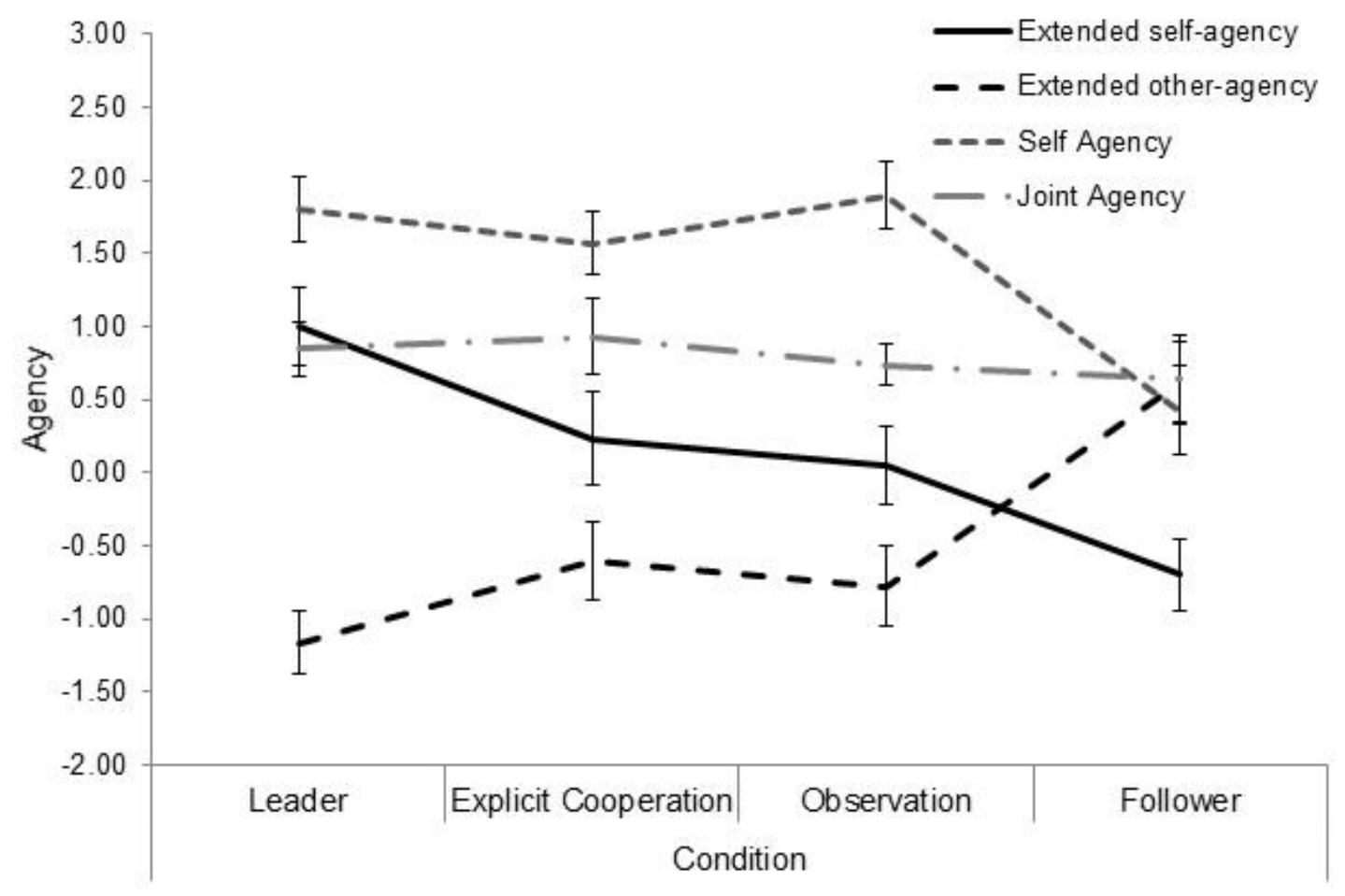

Figure 3. Mean reported agency scores by condition for Experiment 2. Error bars are +/- standard error. 


\section{Supplementary information for \\ Interpersonal synchrony affects performers' sense of agency}

\section{Experiment 1 supplementary results}

\section{Manipulation check}

Participants in the synchrony condition perceived their movements with the other participant as more synchronous $(M=3.93, S D=1.16)$ than in the asynchrony condition $(M=1.66, S D=0.95), t(132)=12.34, p<.001, d=2.13$.

\section{Control variables}

Participants in the synchrony condition reported paying more attention to the other participant during the synchrony manipulation video $(M=3.80, S D=1.44)$ than in the asynchrony condition $(M=2.96, S D=1.50), t(132)=3.32, p=.001, d=0.57$. Although this highlights a potential confound in the experiment, there was no difference between conditions in the reported number of times the cross changed colour, $t(132)=$ $0.60, p=.552, d=0.10-$ an arguably more objective measure of attention. To check that self-reported attention did not significantly impact our results we conducted regression analysis with either extended self-agency or extended other-agency as the dependent variable, and the synchrony manipulation (dummy coded) and self-reported attention as the independent variables. In both cases, the synchrony manipulation remained highly significant $(p<.001)$ and the attention variable was not significant $\left(t_{\max }=0.79, p=.433\right)$.

Twenty-five participants (19\%) selected "the video did not seem like a live stream" in response to the question on connection timing. A further 16 participants indicated some suspicion about the videos being pre-recorded in the open-ended 
questions at the end of the questionnaire. Therefore, a total of $31 \%$ of participants were suspicious about the purported live-stream. Although this might suggest our procedure was not effective at creating the illusion of a live-stream, for those participants' who mentioned a reason for why they thought the video may not be live-stream their reasons did not seem plausible or relevant. For example, one participant wrote: "I think the video was probably not live since the table and room in the video was different from the one that I was in". As the supposed other participant would be in another room it surely would not be surprising that the table and room were different. Instead, it may be participants were primed by the question to think that the video may not be a live stream or it could reflect general suspiciousness about psychology experiments. As one participant mentioned: "I know that the video is not live streaming cos [sic] experiments usually don't do it anyway". To make sure that those participants who were suspicious did not skew the results, we repeated all analysis with just those participants who did not display any suspicion. This did not considerably change any of our results with our main effect of synchrony still highly significant, $F(1,91)=19.02, p<.001, \eta_{\mathrm{p}}{ }^{2}=.17$.

\section{Experiment 2 supplementary results}

\section{Manipulation checks}

A one-way ANOVA found a main-effect of experimental condition for the degree to which participants felt they led the other participant, $F(3,94)=23.29, p<$ $.001, \eta_{\mathrm{p}}{ }^{2}=.43$. Games-Howell post-hoc tests ${ }^{1}$ found that the mean of the leader condition was significantly higher than all other conditions $(p s<.005)$ and the mean of

${ }^{1}$ Due to unequal sample size, Games-Howell tests are used when variance is unequal and Gabriel tests when variance is equal as indicated by Levene's test. 
the follower condition was significantly lower than all other conditions $(p s<.005)$, (see Table S1). A one-way ANOVA also found a main effect of experimental condition for the degree participants felt they followed the other participant, $F(3,94)=21.01, p<$ $.001, \eta_{\mathrm{p}}^{2}=.40$. Critically, Gabriel post-hoc tests showed all group means were lower than the mean for the follower condition $(p s<.01)$. The mean for the leader condition was also lower than the explicit cooperation condition $(p=.002)$, but not the observation condition $(p=.807)$. The explicit cooperation condition was marginally higher than the observation condition $(p=.052)$.

Table S1.

Summary statistics of the manipulation checks for Experiment 2.

\begin{tabular}{lcccc}
\hline & Observation & Exp. Coop & Leader & Follower \\
\hline Led & $2.30(1.63)$ & $2.23(1.83)$ & $4.10(1.43)$ & $0.68(0.70)$ \\
Followed & $2.15(1.61)$ & $3.23(1.40)$ & $1.68(1.36)$ & $4.55(1.17)$ \\
\hline
\end{tabular}

Note. Reported is the mean with standard deviation in parentheses.

\section{Relationships between agency measures}

We examined the correlations between the different measures of agency to explore how they may be related. We first examined the symmetric and asymmetric conditions separately (Table S2) and then examined the leader and follower conditions separately as these conditions were predicted to have divergent effects (Table S3). Notable findings were that extended self-agency was only weakly and negatively correlated with self-agency for the symmetric conditions. This was surprising as logically it might be expected that an expanded sense of self-agency might also entail more certainty over one's own agency. However, there was a strong negative 
correlation between self-agency and extended other-agency for both symmetric and asymmetric conditions. Joint-agency was moderately and significantly correlated with extended self-agency for both the symmetric and asymmetric conditions, but was not significantly correlated with extended other-agency and self-agency. Looking at the two asymmetric conditions separately, joint agency was strongly positively correlated with extended self-agency in the leader condition but not in the follower condition.

Table S2.

Correlation coefficients between the different agency measures for the symmetric conditions (bottom left cells) and asymmetric conditions (top right cells).

\begin{tabular}{lcccc}
\hline & 1 & 2 & 3 & 4 \\
\hline 1. Extended self-agency & & -.17 & $.29 *$ & $.30^{*}$ \\
2. Extended other-agency & $.38^{* *}$ & & $-.47^{* *}$ & .16 \\
3. Self-agency & -.27 & $-.60^{* *}$ & .24 \\
4. Joint-agency & $.33^{*}$ & .13 & -.00 & \\
\hline
\end{tabular}

Note. $* p<.05, * * p<.01$

Table S3.

Correlation coefficients between the different agency measures for the leader condition (bottom left cells) and the follower condition (top right cells).

\begin{tabular}{lcccc}
\hline & 1 & 2 & 3 & 4 \\
\hline 1. Extended self-agency & & 0.23 & -0.06 & 0.13 \\
2. Extended other-agency & 0.15 & & -.22 & 0.24 \\
3. Self-agency & 0.15 & $-.46^{*}$ & & 0.3 \\
4. Joint-agency & $.59 * *$ & 0.25 & 0.04 & \\
\hline
\end{tabular}


Note. $* p<.05, * * p<.01$

\section{Self-other overlap and social bonding measures}

A one-way ANOVA found that there were no significant differences in selfother overlap across conditions, $F(3,94)=1.17, p=.325, \eta_{\mathrm{p}}{ }^{2}=.036$. However, given that all four conditions involved synchrony this was not too surprising.

A one-way ANOVA found a marginally significant main-effect of condition with the combined social bonding measure, $F(3,94)=2.51, p=.063, \eta_{\mathrm{p}}^{2}=.07$. Gabriel post-hoc tests found that the only marginal difference was that the explicit cooperation condition had a higher mean $(M=4.00, S D=1.42)$ than the observation condition $(M=$ 3.10, $S D=1.28), p=.054, d=0.66$. The only variables for which these two conditions differed were in perceived cooperation (see 'control measures' below) and the degree to which they felt they followed the other participant (see 'manipulation check' above). Cooperation was strongly positively correlated with social bonding for these two conditions, $r(46)=.63, p<.001$, whereas perceived following was not, $r(46)=.20, p=$ .166 , possibly indicating that differences in perceived cooperation may play a role in this effect. Social bonding was significantly correlated with extended self-agency, $r(96)$ $=.42, p<.001$, and joint-agency, $r(96)=.50, p<.001$, but not with extended otheragency, $r(96)=.17, p=.090$, or self-agency, $r(96)=-.04, p=.669$.

\section{Control measures}

There was no significant difference across conditions in perceived synchrony, $F(3,94)=0.14, p=.935, \eta_{\mathrm{p}}{ }^{2}<.01$, which was expected as all conditions involved 
synchrony. For the foam pad data we were only able to gather sufficient data from 66 participants due to the malfunctioning of the equipment or participants not pressing hard enough on the foam pad to generate a trigger. To measure synchrony, we calculated the time difference (asynchronies) between consecutive triggers when the triggers were from different pads (i.e., the participant's pad and the confederate's pad). Asynchronies over 1000msec were ignored as these would have been from different beats (e.g., the participant pressed the foam pad last for the previous beat, and the confederate pressed the foam pad first for the next beat). Asynchronies between 500msec-1000msec were retained or deleted on a case by case basis depending on whether they appeared to be due to the participant and the confederate synchronising to the same beat or different beats. We then calculated the mean absolute asynchrony for each participant across conditions. Two participants had extreme scores (over 3SDs from the mean) so were removed (final sample size for each condition was observation $=16$, explicit cooperation $=14$, leader $=14$, follower $=20$ ). There were no significant differences across conditions, $F(3,60)=0.23, p=.875, \eta_{\mathrm{p}}{ }^{2}=.01$, with an overall mean absolute asynchrony of $77.74 \mathrm{msec}(S D=18.07)$. This provides objective data that participants were generally highly synchronised with each other. We also examined the direction of the difference in asynchronies across condition by coding the asynchronies negative when the confederate pressed the pad first and positive when the participant pressed the pad first. There were again no significant differences across conditions, $F(3,60)=0.98$, $p=.406,, \eta_{\mathrm{p}}^{2}=.05$.

One-way ANOVAs revealed no significant differences across conditions with the control variables of mood valence $\left(p=.127, \eta_{\mathrm{p}}^{2}=.06\right)$, mood arousal $\left(p=.272, \eta_{\mathrm{p}}{ }^{2}=\right.$ $.04)$, how enjoyable the task was $\left(p=.204, \eta_{\mathrm{p}}{ }^{2}=.05\right)$, perceived success of one's own performance $\left(p=.837, \eta_{\mathrm{p}}^{2}=.01\right)$, perceived success of the other participant's 
performance $\left(p=.338, \eta_{\mathrm{p}}{ }^{2}=.04\right)$, perceived success at working together $\left(p=.113, \eta_{\mathrm{p}}{ }^{2}=\right.$ $.06)$, and prediction $\left(p=.223, \eta_{\mathrm{p}}{ }^{2}=.05\right)$. There was a significant difference across conditions in handedness, $F(3,93)=3.50, p=.018, \eta_{\mathrm{p}}{ }^{2}=.10$. This was primarily due to three predominantly left-handed participants in the leader condition. Removal of these participants and one participant who did not answer the question did influence some of the marginally significant differences, in-part due to the loss of power. For example, the difference in extended self-agency between the leader condition and the symmetric conditions became no longer significant ( $p=.129$, with Bonferroni correction). However, other key highly significant findings were not affected. We did find significant differences with attention, $F(3,94)=4.60, p=.005, \eta_{\mathrm{p}}^{2}=.13$, with GamesHowell post-hoc test showing the follower condition had a higher mean than the leader condition $(p=.008, d=0.96)$ and the observation condition $(p=.003, d=1.04)$. The conditions differed in perceived cooperation, $F(3,94)=4.22, p=.008, \eta_{\mathrm{p}}^{2}=.12$, but Gabriel post-hoc test showed that the only difference was the explicit cooperation condition was higher than the observation condition $(p=.007, d=1.01)$. There was also significant differences with perceived difficulty, $F(3,94)=3.80, p=.013, \eta_{\mathrm{p}}{ }^{2}=.11$, although Games-Howell post-hoc tests only showed marginal differences, with the follower condition having a higher mean than the observation condition $(p=.062, d=$ $0.73)$ and the explicit cooperation condition $(p=.061, d=0.74)$.

We conducted multiple regressions to check that it was our manipulation of role that produced the effects we found on extended self-agency, extended other-agency, and self-agency, and not these confounding factors. Because our significant differences in extended self-agency, extended other-agency, and self-agency occurred with the leader and follower conditions, we only included a dummy variable comparing these two conditions and we only focused on the control variables of attention and difficulty as a 
significant difference was only found between the observation and explicit cooperation condition for cooperation. A regression with extended self-agency as the dependent variable found that neither attention nor difficulty were significant, only the dummy variable of follower or leader condition (see Table S4); likewise with extended otheragency. For self-agency, perceived difficulty was a significant predictor, but it was not as strong a predictor as the condition dummy variable. In summary, although selfreported attention, cooperation, and difficulty, also varied between conditions suggesting that these variables may have impacted our results, our manipulation was still the best predictor of the differences in our agency measures.

\section{Table S4.}

Regression coefficients for the predictors of condition, attention, and difficulty, for three regressions with the dependent variables of extended self-agency, extended otheragency, and self-agency.

95\% Confidence interval

Standardised

coefficient

Lower Upper

limit limit

Extended self-agency

$\begin{array}{llllll}\text { Condition } & -0.46 & -3.47 & .001 & -2.24 & -0.59 \\ \text { Attention } & -0.14 & -1.09 & .283 & -0.46 & 0.14 \\ \text { Difficulty } & -0.21 & -1.75 & .088 & -0.53 & 0.04\end{array}$

Extended other-agency

$\begin{array}{llllll}\text { Condition } & 0.51 & 3.63 & .001 & 0.75 & 2.63 \\ \text { Attention } & 0.06 & 0.46 & .648 & -0.27 & 0.42\end{array}$


Difficulty

$-0.02$

$-0.18$

.858

$-0.35$

0.30

Self-agency

Condition

$-0.41$

$-2.99$

.004

$-1.85$

$-0.36$

Attention

$-0.13$

$-0.97$

.336

$-0.41$

0.14

Difficulty

$-0.26$

$-2.17$

$.036 \quad-0.53$

$-0.02$

Note. Condition is leader (dummy code $=0$ ) versus follower (dummy code $=1$ ). 